\title{
Effet de la réutilisation des eaux partiellement usées sur l'économie
}

\author{
M.A. Komiha \\ E.N.S.E.T. de Tunis, 5, Avenue Taha Hussein, 1008 Montfleury - Tunisie
}

\section{Introduction}

Il est dangereux pour un pays en voie de développement de croire qu'il peut se développer sans disposer d'une quantité d'eau suffisante, ou s'il en dispose, de penser que cela suffit à assurer la croissance de la population et de l'économie. Si l'augmentation de la consommation de l'eau potable en Tunisie suit l'évolution démographique actuelle, un manque considérable en eau se produira à l'horizon 2000 , en raison des faibles potentialités hydrauliques actuelles du pays. Donc il est bien temps d'adopter une stratégie d'économie d'eau disponible et d'augmenter les ressources d'eau afin de répondre correctement aux besoins en eau dans les prochaines années.

\section{Potentiel hydraulique}

Le milieu naturel tunisien est réputé difficile. En effet, le potentiel hydraulique naturel (P.H.N.) ou utilisable de notre pays est le plus faible par rapport à ses voisins (P.H.N. du Maroc est estimé à $350 \mathrm{Mm}^{3} / \mathrm{an}$ ). La Tunisie est comprise entre la zone méditerranéenne semi-aride et la zone saharienne, d'oủ la faiblesse des ressources en eaux permanentes. La pluviométrie est très irrégulière aussi bien dans le temps que dans l'espace. Elle varie de $1200 \mathrm{~mm} / \mathrm{an}$ à l'extrême nord à $100 \mathrm{~mm} / \mathrm{an}$ au sud et elle déverse annuellement sur l'ensemble du territoire national $33 \mathrm{Mm}^{3}$ d'eaux. Signalons que la demande bioclimatique en eau (évapotranspiration) est très élevée : 1300 à $1500 \mathrm{~mm} / \mathrm{an}$, avec un maximum de 6 à $8 \mathrm{~mm} / \mathrm{j}$ pendant les mois de pointe (juillet, août).

Les ressources potentielles conventionnelles en eau sont évaluées à $4,5 \mathrm{Mm}^{3} / \mathrm{an}$ (14\% de la pluviométrie) réparties en :

- 60,2 \% en eaux de surface dont $51,7 \%$ sont exploitables par un ensemble d'ouvrages comportant 18 barrages et 64 barrages et lacs collinaires (dont 42 en cours de mise en eau).

- $39,8 \%$ en eaux souterraines, les deux tiers proviennent des nappes profondes dont $74,7 \%$ sont exploitables à partir d'un ensemble de 1830 forages de plus de 2500 mètres de profondeur et 89 sources et le tiers provient des nappes phréatiques dont $97,2 \%$ sont exploitables à partir des 99000 puits de surface.

\section{Répartition géographique des ressources}

Ces ressources sont inégalement réparties sur l'ensemble du pays. Une simple comparaison entre les régions illustre les disparités dans ce domaine :

- le Nord détient près de $60 \%$ du potentiel en eau du pays, répartis aux deux tiers au Nord-Ouest. Ces ressources en eau sont constituées pour plus des trois quarts par les eaux de surface. Les deux tiers sont exploités pour $40 \%$ de la population du pays et moins de un cinquième du territoire national ;

- le Centre détient près de $17 \%$ du potentiel en eau du pays répartis aux cinq sizième au Centre-Ouest. Ces ressources en eau sont contituées de prédominance (trois quarts) des eaux souterraines dont les deux-tiers sont exploités pour seulement le tiers de la population du pays et le quart du territoire national ;

- le Sud détient près du quart du potentiel en eau du pays réparti aux deux tiers au Sud-Ouest. Ces ressources en eaux sont constituées essentiellement par des eaux souterraines (nappes profondes) exploitables à moitié, en raison de la crainte d'augmentation du degré de salinité, réservées pour $15,3 \%$ de la population du pays installée sur $62 \%$ du territoire national dont le quart est formé de zones désertiques.

Justifié par les disparités existantes entre les ressources de l'intérieur et les besoins croissants du littoral, le principe de vastes transferts inter-régionaux a été admis et appliqué dans la mise en œuvre des trois plans directeurs des eaux couvrant chacune des régions du Nord, du Centre et du Sud. C'est ainsi que grâce à la souplesse de transfert des eaux que le Nord subsiste à ses besoins croissants en eau potable pour assurer le développement normal de ses régions, mais le Centre est déjà déficitaire en eau car il ne pourrait vivre sans les transferts importants d'eau en provenance du Centre-Ouest, par contre le Sud arrive à son autonomie en quantité d'eau en provenance essentielle des nappes côtières et transfert à partir de forage des nappes sahariennes de l'extrême Sud.

Il ressort de l'analyse de la situation des projets que si l'augmentation de la consommation de l'eau en Tunisie suit l'évolution démographique et le développement des secteurs agricoles industriels et touristiques, un manque quantitatif considérable en eau se produira à l'horizon 2000 , en raison des faibles et fragiles potentialités hydrau- 
liques actuelles du pays. C'est pour cette raison, qu'il convient de souligner l'importance de la gestion de l'eau. Donc, il est bien temps d'adopter une stratégie d'augmentation des ressources d'eau et de sa disponibilité à l'échelle nationale afin de répondre correctement aux besoins futurs en eau. L'utilisation rationnelle de l'eau présente aussi une grande importance.

\section{Prévision des besoins futurs en eau}

Les prévisions futures des besoins en eau dépendent essentiellement des facteurs énumérés ci-après suivant l'ordre d'importance :

- l'évolution démographique et de la croissance urbaine ;

- la croissance de l'agriculture ;

- le développement industriel ;

- la croissance du tourisme.

\section{Evolution démographique de la Tunisie}

Après l'indépendance, la Tunisie a subi d'importantes transformations (croissance démographique, mutations économiques, changement des modes de vie), qui se répercutent inévitablement sur l'organisation de l'espace: infrastructures et équipements nouveaux. Support d'une société toujours plus complexe, dont les relations avec l'environnement sont toujours plus interdépendantes, le territoire national s'impose désormais comme une ressource limitée et souvent fragile, dont l'utilisation optimum (exploitation des potentialités offertes, préservation du patrimoine naturel) nécessite une action d'ensemble.

Lorsqu'on examine une carte démographique de Tunisie, nous sommes frappés par le déséquilibre du peuplement. Le territoire reste profondément dominé par la puissance démographique de la capitale. Plus d'un million d'habitants, un huitième de la population nationale, s'entasse dans moins de $1 \%$ de la superficie du territoire national. Le rapport entre la densité de la population de la région de la capitale, la plus peuplée, et celle de la région la moins peuplée, est de l'ordre de 1 à 117 .

Le peuplement s'est en général développé dans les régions les plus riches en eau se trouvant au nord de la Tunisie. Il reste que dans les autres parties du pays, c'està-dire dans $60 \%$ du territoire, le niveau des densités peut aller jusqu'à $25 \%$ de la moyenne nationale.

\section{Les perspectives d'évolution démographique}

Les perspectives d'évolution démographique ont justifié l'élaboration de deux scénarios contrastes d'évolution, destinés à mettre en évidence les options essentielles qui doivent fonder la conception du schéma national :

- un scénario A dit d'équilibre, qui suppose que chaque région absorbe son accroissement naturel de la population et que les mouvements migratoires s'annulent ou se compensent entre les régions ;

- un scénario B dit de concentration, où il est supposé que la population rurale s'accroîtrait malgré tout de $10 \%$ dans toutes les régions, mais que la moitié de l'accroissement naturel des régions de l'Ouest et du Sud se reportait vers les centres urbains du Nord-Est et du Centre-Est, ce qui représenterait l'émigration de près de deux millions d'habitants à l'an 2000.

Ces deux scénarios ne constituent pas des variantes extrêmes, mais des évolutions plausibles dans le cadre d'une politique d'aménagement du territoire recherchant un équilibre acceptable. Bien qu'amplifiant la tendance actuelle, il s'agit d'une augmentation démographique de penchant de concentration modérée (voir la figure l).

\section{Besoins futurs en eau en l'an 2000}

Malgré que les besoins en eau de la région du Nord sont devenus très importants de prédominance agricole $80 \%$, et un transfert de quantité d'eau aux régions côtières multiplié par dix par rapport au transfert actuel, cette région connaîtra une autonomie en eau grâce à la souplesse de transfert des ressources en eau.

Par contre, le Centre et le Sud connaîtront une augmentation considérable des besoins en eau d'environ $80 \%$. Cette consommation prévisible dépasse de loin le total des ressources en eau mobilisables. Donc l'augmentation des besoins sera partiellement satisfaisante par une exploitation accrue de la nappe Continentale Intercalaire, nécessitant la multiplication des forages à grande profondeur ce qui engendrera des dépenses énormes à réaliser avec ce qui comportera comme risque d'augmentation du degré de salinité.

L'accroissement des ressources existantes est très coûteux parce que nous avons déjà épuisé la plupart des solutions faciles et économiques. Le gouvernement tunisien prévoit pour cela la construction d'un ensemble d'ouvrages hydrauliques comportant : 21 barrages, 202 barrages collinaires, 610 puits profonds et 1000 lacs collinaires apportant un volume théorique annuel supplémentaire en eau respectivement de $720 \mathrm{Mm}^{3}, 110 \mathrm{Mm}^{3} 288 \mathrm{Mm}^{3}$ et 2000 $\mathrm{Mm}^{3}$. En plus, les deux sources envisageables pour reculer les limites des ressources disponibles - dessalement des eaux de mer et saumâtres, et le recyclage des eaux usées sont techniquement faisables, mais à des coûts excessivement élevés. Or il n'est pas possible d'augmenter rapidement la quantité d'eau du jour au lendemain, donc il faut appliqué rigoureusement des stratégies visant à économiser l'eau et à limiter le gaspillage.

La première stratégie à envisager est donc de maintenir la consommation d'eau à un niveau minimal. La conservation est la pierre angulaire de la maîtrise de la demande en eau. Elle requiert une meilleure utilisation de l'eau disponible. La conservation de l'eau peut prendre plusieurs formes allant de la position que peut prendre un individu à la position de l'Etat. 


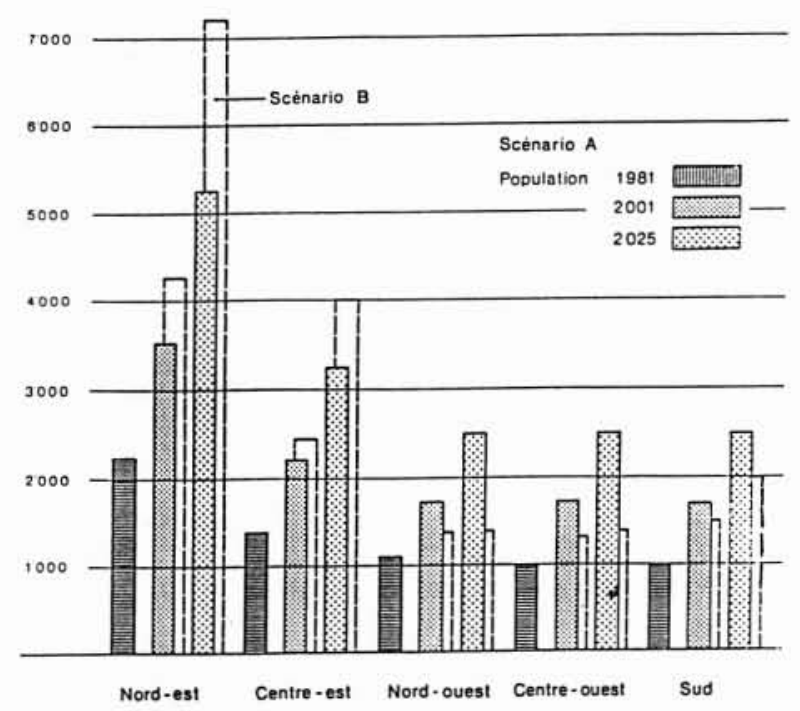

1. Evolution démographique du pays.

\section{Expérience de la cité universitaire de Montfleury}

\subsection{Présentation de la cité}

La cité universitaire se situe dans la zone de Montfleury, 5 Avenue Taha Hussein, dans un quartier de haut standing où le coefficient d'occupation de sol est égal à 1 , de ce fait les eaux usées sont directement évacuées vers le réseau de l'Office national de l'assainissement (O.N.As.).

La cité universitaire est subdivisée en deux parties comme suit :

- restaurant universitaire; placé au rez-de-chaussée du bâtiment fournissant près de 2500 repas par jour ouvrable, une lingerie qui nettoie l'ensemble des fournitures du foyer, et une administration complète de la cité ;

- foyer universitaire ; qui se compose de sept étages identiques comprenant chacun :

* 30 chambres identiques réservées pour deux étudiants chacune avec deux lavabos.

* 2 blocs sanitaires, placés aux deux extrémités de l'étage. Le premier bloc qui est placé en face de l'escalier comprend 3 toilettes, 3 douches et 2 lavabos, et le second qui est placé à côté de l'escalier comprend 3 toilettes et 2 lavabos. Ce qui nous donne en totalité, pour le foyer, un ensemble de 210 chambres, 476 lavabos, 42 douches et 72 toilettes pour 420 étudiants.

\subsection{Consommation d'eau potable}

Nous remarquons que suivant les statistiques, la consommation moyenne annuelle (C.M.A.) en eau de la cité est estimée à $43850 \mathrm{~m}^{3}$ d'eau répartis respectivement suivant les quatre trimestres de l'année en $30 \%, 22,5 \%, 23 \%$ et $24,5 \%$. La statistique entreprise pendant deux années de suite nous a donné les résultats des consommations moyennes suivantes :

- $120 \mathrm{l} / \mathrm{j} /$ douche, ce qui donne $3,6 \mathrm{~m}^{3} / \mathrm{an} /$ étudiant ; - $119 \mathrm{l} / \mathrm{j} /$ toilette WC, ce qui donne $6,12 \mathrm{~m}^{3} /$ an/étudiant ; - $156 \mathrm{l} / \mathrm{j} / \mathrm{d}$ 'eau froide et $500 \mathrm{l} / \mathrm{j}$ d'eau chaude pour la lingerie, ce qui donne en totalité $0,47 \mathrm{~m}^{3} /$ an/étudiant ;

- $950 \mathrm{l} / \mathrm{j}$ pour le lavage de légumes, ce qui donne 0,12 $\mathrm{m}^{3} /$ repas ;

- $2000 \mathrm{l} / \mathrm{j}$ pour le nettoyage de carrelages du restaurant, ce qui donne $600 \mathrm{~m}^{3} / \mathrm{an}$.

\subsection{Système d'évacuation des eaux usées}

Il est basé sur le système pseudo-séparatif (évacuation séparée des eaux de buanderie et les eaux usées) :

- dans chaque chambre, les lavabos sont reliés à une conduite commune traversant les chambres verticalement du $7^{e}$ étage jusqu'au premier, puis ils sont reliés aux regards placés au rez-de-chaussée ;

- dans les cabinets des douches et toilettes (WC), une conduite évacue les eaux des douches en passant d'un étage à un autre. Mais les toilettes (WC) évacuent verticalement les eaux collectées dans un regard au R.D.C.;

- la lingerie évacue les eaux usées directement vers les regard placé au R.D.C. ;

- le restaurant possède un réseau d'évacuation des eaux usées indépendant des autres.

\subsection{Méthodologie de la R.E.P.U.}

\section{a) Système utilisé}

Il ressort de cette analyse que nous pouvons réaliser un gain quantitatif d'eau de la façon suivante:

- dans le foyer, nous pouvons utiliser les eaux usées évacuées par les douches pour les chasses d'eau des toilettes (WC) de l'étage de dessous après un simple traitement; - dans le restaurant, nous pouvons utiliser les eaux usées évacuées par la lingerie du foyer et le lavage des légumes pour le nettoyage du carrelage du restaurant et ceci après stockage et traitement.

\section{b) Analyses des eaux usées non traitées}

Nous avons obtenu les résultats présentés ci-après suivant les analyses réalisées à l'Institut Pasteur de Tunis :

- les eaux usées des douches sont des eaux savonneuse (5 à $7,5 / \mathrm{g} 100 \mathrm{ml}$ ) ayant une faible quantité de graisse et sans la présence des germes totaux (à $37^{\circ}$ ), mais vu l'excès des coliformes totaux et fécaux, l'eau est donc impropre à la consommation. Par contre nous pouvons utiliser ces eaux usées dans les chasses d'eau des toilettes;

- les eaux usées de la lingerie sont des eaux chargées de détergents, riches en germes de pollution fécale. Les coliformes totaux sont supérieurs à $1200 \mathrm{~g} / 100 \mathrm{ml}$ et de streptocoques fécaux sont inférieurs à $3 \mathrm{~g} / 100 \mathrm{ml}$. L'eau est donc impropre à la consommation, mais nous pouvons 
utiliser ces eaux usées comme une eau de seconde main après traitement pour le lavage de carrelage du restaurant ; - les eaux usées de lavage des légumes sont polluées, riches en microorganismes pathogènes et en streptocoques fécaux dont la concentration est supérieure à $23 \mathrm{~g} / 100 \mathrm{ml}$. L'eau est donc impropre à la consommation, mais nous pouvons utiliser ces eaux pour le nettoyage des carrelages du restaurant après un traitement.

\section{c) Analyses des eaux usées traitées}

Suivant les analyses réalisées à l'Institut de Pasteur - Tunis - des eaux usées traitées par simple filtration dans le sable de différentes granulométries, nous avons obtenu les résultats ci-après :

- Les eaux usées des douches n'ont pas besoin de traitement poussé car le système d'économie d'eau ne permet pas de contact avec l'être humain. Par contre, il faut vérifier l'état des conduites placées entre le réservoir et les chasses d'eau des toilettes (WC).

- Nous remarquons que les caractéristiques bactériologiques des eaux usées de la lingerie et de lavage des légumes n'ont pas changé et que ces eaux sont toujours impropres à la consommation. Par contre il y a toujours la possibilité de les utiliser pour le nettoyage des carrelages du restaurant en prenant des précautions d'hygiène (exp : des gants obligatoires pour les ouvriers).

\subsection{Moyen d'économie}

\section{a) Au foyer}

Il faut réaliser des réservoirs de capacité au moins égale à la consommation journalière des 3 chasses d'eau des toilettes, ce qui nous donne un volume égal à $0,45 \mathrm{~m}^{3}$. Nous avons réalisé une cellule expérimentale (réservoir) qui fonctionne jusqu'à nos jours. Nous réaliserons un gain quantitatif annuel d'eau potable évalué à $2160 \mathrm{~m}^{3}$ si tout le système est implanté au foyer.

\section{b) Au restaurant}

Nous pouvons réaliser un réservoir souterrain placé juste à côté du restaurant d'une capacité d'au moins égale à la consommation réservée au nettoyage du carrelage du restaurant sans oublier le système de traitement et de pompage.

\subsection{Micro-économie de l'étude}

Nous pouvons économiser annuellement suivant les statistiques faites $1562 \mathrm{~m}^{3}$ (au foyer $1080 \mathrm{~m}^{3}$ et au restaurant $482 \mathrm{~m}^{3}$ ), ce qui représente un coût estimé suivant la tarification actuelle de l'eau de 1368 Dinars Tunisiens (D.T.). Le coût de l'installation entière du système d'économie est estimé à 3100 D.T.

En tenant compte d'un coût supplémentaire annuel d'entretien et de réparation de l'installation du système d'économie évalué à $10 \%$ du coût du système d'économie, l'amortissement des investissements est possible en deux ans en tenant compte du prix actuel de l'eau. Cette durée pourrait être réduite si le prix unitaire de l'eau continue à augmenter comme auparavant.

\subsection{Marché et avenir de l'étude}

Les premières investigations sont très encourageantes car nous pourrons passer de la micro-économie de l'étude à la macro-économie car il suffit de généraliser l'implantation de ce procédé à toute construction étatique ou privée en Tunisie.

Sachant qu'en Tunisie, nous avons 65 foyers réservés pour 30000 étudiants (au nord 38 foyers et 18000 étudiants, au centre 16 foyers et 7000 étudiants et au sud 11 foyers et 4500 étudiants). Si nous appliquons seulement la méthode de l'utilisation des eaux usées des douches comme eau de seconde main, nous pourrons économiser annuellement $108000 \mathrm{~m}^{3}$.

D'autres applications sont projetées comme le secteur touristique qui rencontre des problèmes de plus en plus importants relatifs à la quantité d'eau pendant la période estivale. Devant les consommations élevées constatées dans les unités hôtelières et touristiques, il a été entrepris des investigations sur les origines de ces consommations et la manière de les réduire, sans pour autant influer sur la qualité de service.

\section{Conclusions}

En raison des faibles potentialités hydrauliques actuelles du pays, nous sommes appelés plus que jamais à adopter une stratégie en vue d'économiser l'eau disponible. Parmi les procédés d'économie de l'eau connus, la réutilisation des eaux usées après un simple traitement constituerait une nouvelle ressource additionnelle et économique en eau. L'expérience menée à la Cité universitaire de Montfleury - Tunis - basée sur un procédé de traitement simple et économique, permet la réutilisation des eaux partiellement usées. L'essai pourrait permettre d'économiser :

- au foyer: $1080 \mathrm{~m}^{3}$ par an d'eaux usées de douches pour les chasses d'eau des toilettes de l'étage de dessous ;

— au restaurant : $482 \mathrm{~m}^{3}$ par an d'eaux usées de la lingerie et du lavage des légumes pour le nettoyage des carrelages du restaurant.

Ceci représente une économie non négligeable, d'autant plus que nous généraliserons l'implantation de ce système d'économie à toutes les cités universitaires et les hôtels en appliquant rigoureusement les recommandations concernant l'hygiène. Cette économie constituerait une nouvelle ressource additionnelle en eau et permet de reculer les limites des ressources disponibles actuellement. 